\title{
AOR
}

Selected Papers of \#AoIR2020:

The $21^{\text {st }}$ Annual Conference of the

Association of Internet Researchers

Virtual Event / 27-31 October 2020

\section{EXTENDING GAMING DEMAND: SUBJECTIVE EXPERIENCES OF COGNITIVE, EMOTIONAL, PHYSICAL, AND SOCIAL VIDEOGAMING REQUIREMENTS}

\author{
Nick Bowman \\ Texas Tech University \\ Jaime Banks \\ Texas Tech University
}

Videogames involve players as co-creators of on-screen events (Wellenreiter, 2015) and this interactivity drives enjoyment (Grodal, 2000). Although interactivity is inherent to videogames, it is variably perceived by users (Stromer-Galley, 2004). Through both inherent and perceived involvement, interactivity is best considered a demanding process taxing players' limited attentional resources (Fisher et al., 2018).

Videogaming demand includes at least four dimensions (Bowman et al., 2018): cognitive (processing of game logics/tasks), emotional (affective responses to game events/outcomes), physical (managing controller inputs/interfaces), and social (responding to human/nonhuman actors). These dimensions have been validated through quantitative empirical data analysis as the Video Game Demand Scale (VGDS, Bowman et al. 2018). However, a critical limitation of theory-driven scale instruments is their predetermined meaning of a construct. However, people may experience a phenomenon in ways not accounted for in a metric's grounding theory. Toward better understanding demand phenomenology as it unfolds in the player-game relation (cf. Vargas-Iglesias \& Navarrete-Cardero, 2019), we explore (RQ1) What factors are prevalent in gamers' recollections of videogaming demand?

\section{Method}

We performed secondary analysis of open-ended participant data made publicly available by Bowman et al. (2018; https://osf.io/x5jch/, with permission). The original study comprised gamers (a) writing essays of recent gaming experiences and (b) writing Suggested Citation (APA): Bowman, N. D., \& Banks, J. (2020, October 28-31). Extending gaming demand: Subjective experiences of cognitive, emotional, physical, and social videogaming requirements. Paper presented at AoIR 2020: The 21 $1^{\text {th }}$ Annual Conference of the Association of Internet Researchers. Virtual Event : AolR. Retrieved from http://spir.aoir.org. 
short essays on "how [cognitively/emotionally/physically/socially] demanding" that experience was (Table 1), as (c) well evaluating the videogame experience on various dimensions (including VGDS; those analyses published by the original authors). The published dataset included $N=660$ participants (demographic details reported in Bowman et al., 2018).

\section{Table 1.}

Descriptive results of participants' open-ended demand responses.

\begin{tabular}{lcccc}
\hline & $\begin{array}{c}\text { \# of } \\
\text { responses }\end{array}$ & $\begin{array}{c}\text { Word count } \\
M(S D)\end{array}$ & $\begin{array}{c}95 \% \mathrm{Cl} \\
\text { lower | upper }\end{array}$ & $\begin{array}{c}\text { Word count } \\
\text { Median }\end{array}$ \\
Cognitive demand & 629 & $41.65 \mathrm{a}(30.30)$ & $39.28 \mid 44.02$ & 33 \\
Emotional demand & 608 & $38.24 \mathrm{a}(26.44)$ & $36.14 \mid 40.34$ & 33 \\
Physical demand & 611 & $24.64 \mathrm{~b}(18.54)$ & $23.17 \mid 26.11$ & 19 \\
Social demand & 601 & $40.30_{\mathrm{a}}(29.45)$ & $37.95 \mid 42.65$ & 33 \\
\hline
\end{tabular}

Note: Subscripts indicate means that differ significantly from each other $(p \leq .05)$.

The demand-recall essays were subjected to semantic network analysis to identify themes (i.e., induced word clusters) using Leximancer 4.51

(https://info.leximancer.com/; Smith \& Humphreys, 2006). A complete accounting of the analysis (including data processing, software settings, data/output files, and analysis narrative) is available online: https://osf.io/fv7jt.

\section{Results}

Induced themes are presented here in brief, with more granular details in the linked supplements; findings are also visually summarized in Figure 1.

\section{Figure 1.}

Semantic network analysis of player's discussion of videogame demands. Cognitive Demands Emotional Demands
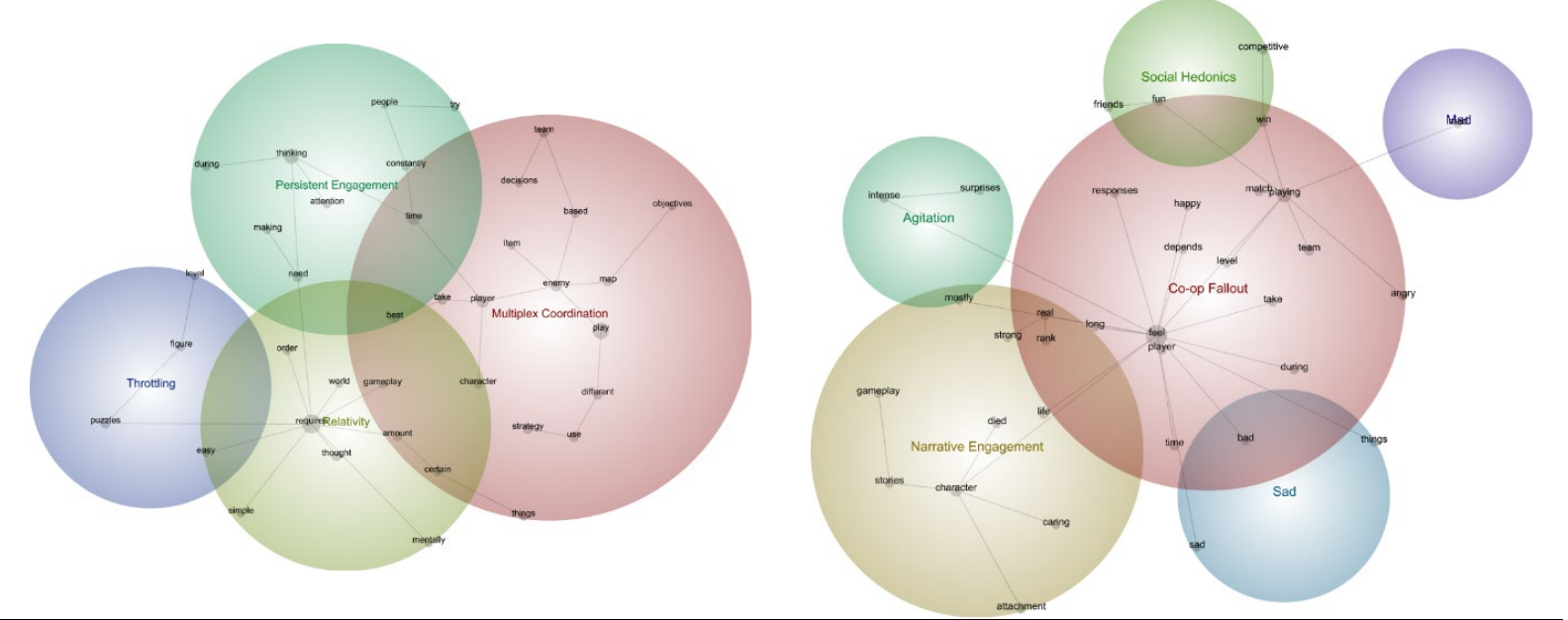


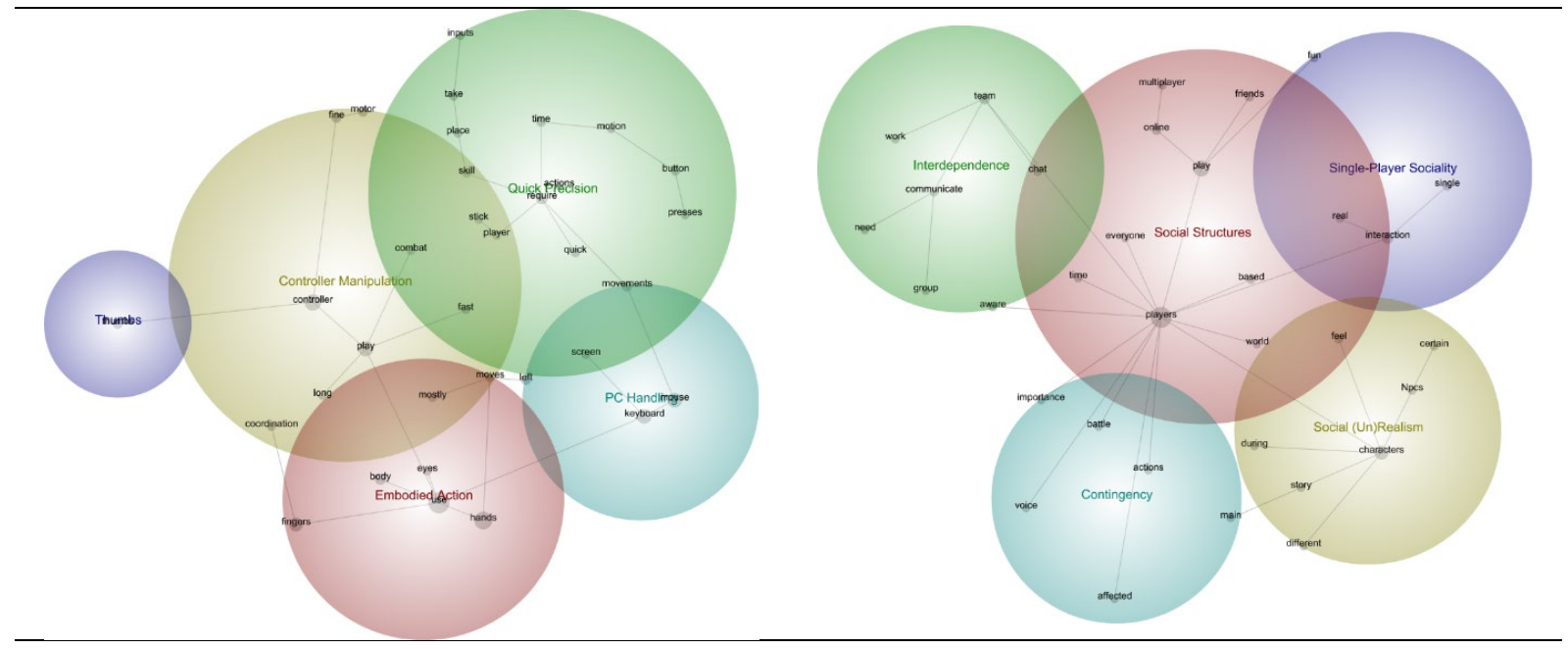

Note: Full-sized images are available in the linked supplemental files for this abstract.

For cognitive demand, four themes emerged: coordination, requirements to enact strategies based on dynamic in-game information sources; persistent engagement, requirements to constantly monitor and organize information; throttling, making deliberate decisions to control how much thought-effort is made toward desired outcomes; relativity, awareness of in-game challenges in relation to the game's design.

For emotional demand, five themes emerged: cooperative fallout, reactions to stressors of playing with others; narrative engagement, attachment to or investment in unfolding stories; social hedonics, expressing joy of co-playing (especially victories); agitation, reactions to surprising disruptions of gameplay events. Surprisingly, two discrete emotions emerged as distinct themes: anger (usually when losing) and sadness (generally at in-game narrative turns).

For physical demand, five themes emerged: embodied action, discrete body movements often focused on eye-hand coordination; controller manipulation, engaging handheld devices; quick precision, quick input and controller response relative to eventdriven player needs. Discrete themes were also present for familiar physical control modes: $P C$ controls such as keyboard-and-mouse setups, and thumbs in reference to manipulating handheld controllers.

For social demand, five themes emerged: social structures, interpersonal/group communication dynamics related to the game; social (un)realism, non-human characters and considerations of their perceived (lack of) "humanness;" interdependence, the necessity of coordination for co-playing; single-player sociality, characterizations of playing with others compared to playing alone; and contingency, the interdependence dynamics of in-game social dialogue.

\section{Discussion}

Current explications of videogaming demand (such as those informing VGDS; Bowman et al., 2018) rely on theoretical suppositions to define the cognitive, emotional, physical, and social demands, which may not necessarily engage all possible player perceptions 
of what games require of them during play. Findings of the present study support previously theorized demand dynamics, but also indicate nuances not unpacked in those theory-driven analyses.

With cognitive demands, our analysis largely comports with the original work, as players were primarily concerned with mental efforts associated with processing and making sense of in-game information-largely ludic concerns. Similarly, the present findings for physical demand aligned largely with original operationalizations, as players were keenly concerned with the fine-motor skills and tangible controller efficiencies required to engage videogames (the "golden hands" heuristic; see Parisi, 2015).

Player experiences diverged more noticeably for emotional and social demands. Concerning emotional demands, analysis supports the focus on eudaimonic, narrativedriven emotional states (Oliver et al., 2015). In addition, more basic emotional demand (missed in original theorizing) also emerged: players reported intense, discrete, emotional "highs and lows" tied to gameplay performance, often magnified in the presence of others. For social demands, this analysis revealed a compelling departure from VGDS. When players recalled human gaming partners, social demand corresponded mostly with interpersonal and organizational communication characteristics: interdependence dynamics, negotiating personalities, negotiating roles. When players discussed game-character partners, they talked more about feeling or lacking a "human-like" connections with them. A critical take-away here is that, although both human and non-human others are socially demanding, the ways in which that demand is felt by players seems categorically different.

Future work would benefit by focusing on in situ videogame play, as well as a more granular analysis of demands as a function of game genre or content. Gamer experience as well as social identification as "a gamer" might also influence felt demands, as well as other cultural, demographic, and psychographic factors relevant to gaming preference, playstyle, and performance.

\section{References}

Bowman, N. D., Wasserman, J., \& Banks, J. (2018). Development of the Video Game Demand Scale. In N. D. Bowman (Ed.), Video games: A medium that demands our attention (pp. 208-233). Routledge.

Fisher, J.T., Huskey, R., Keene, J.R., \& Weber, R. (2018). The limited capacity model of motivated mediated message processing: Looking to the future. Annals of the International Communication Association, 42(4), 291-315.

Grodal, T. (2000). Video games and the pleasures of control. In D. Zillmann \& P. Vorderer (Eds.), Media entertainment: The psychology of its appeal (pp. 197-213). LEA.

Oliver, M. B., Bowman, N. D., Woolley, J. K., Rogers, R., Sherrick, B., \& Chung, M-Y. (2015). Video games as meaningful entertainment experiences. Psychology of Popular Media and Culture, 5(4), 390-405. 
Parisi, D. (2015), A counterrevolution in the hands: The console controller as an ergonomic branding mechanism. Journal of Games Criticism, 2(1).

Smith, A.E., \& Humpreys, M.S. (2006). Evaluation of unsupervised semantic mapping of natural language with Leximancer concept mapping. Behavioral Research Methods, 38, 262-279.

Stromer-Galley, J. (2004). Interactivity-as-product and interactivity-as-process. The Information Society, 20(5). 391-394.

Toh, W. (2019). A multimodal approach to video games and the player experience. Routledge.

Vargas-Iglesias, J.J., \& Navarrete-Cardero, L. (2019). Beyond rules and mechanics: A different approach for ludology. Games and Culture. 